\title{
Robótica educativa para enseñar matemáticas: opiniones de los estudiantes del Grado en Educación Primaria
}

\author{
Luis Manuel Soto-Ardila ${ }^{1}$, Lina Melo Niño ${ }^{2}$ y Ana Caballero Carrasco ${ }^{3}$ \\ ${ }^{1}$ Departamento de Didáctica de las Ciencias Experimentales y Matemáticas de la \\ Universidad de Extremadura, España. | luismanuel@unex.es | https://orcid.org/0000-0002- \\ 1041-4077 \\ 2 Departamento de Didáctica de las Ciencias Experimentales y Matemáticas de la \\ Universidad de Extremadura, España. | Ivmelo@unex.es | https://orcid.org/0000-0003-4771- \\ 058X \\ ${ }^{3}$ Departamento de Didáctica de las Ciencias Experimentales y Matemáticas de la \\ Universidad de Extremadura, España. | acabcar@unex.es | https://orcid.org/0000-0001- \\ 7538-3833
}

\begin{abstract}
Resumen: A lo largo de los años, la robótica educativa ha ido ganando más peso, estando cada vez más presente en el proceso de enseñanza y aprendizaje. El uso de la robótica como recurso educativo es importante por los beneficios que puede ocasionar entre los alumnos, como puede ser aumento de atención, motivación, curiosidad, etc., tal y como puede observarse en los diferentes estudios analizados para esta investigación. Cada vez son más los autores que estudian los beneficios o perjuicios que la robótica educativa puede tener en el proceso de enseñanza y aprendizaje de los alumnos. La presente investigación se encargará de analizar las opiniones de los futuros maestros de primaria sobre el uso de la robótica como recurso educativo para enseñar matemáticas. Se analizarán las opiniones de cincuenta estudiantes del Grado de Educación Primaria de la Universidad de Extremadura (Badajoz, España). Los datos se obtienen mediante una pregunta abierta a través de un foro y, para poder realizar los análisis pertinentes, se recurrió al software WebQDA (Neri, Costa y Moreira, 2011). Con WebQDA se codificaron las distintas opiniones de los estudiantes, según un sistema de categorías diseñado por Soto-Ardila, Caballero, Melo y Luengo (2019). Los resultados muestran que, para la mayoría de los alumnos de la muestra, el uso de la robótica puede otorgar numerosos beneficios en el proceso de enseñanza-aprendizaje en general y, más concretamente, en la enseñanza y aprendizaje de las matemáticas, siendo estos beneficios, mayores que las desventajas que su uso puede ocasionar.
\end{abstract}

Palabras clave: Robótica Educativa; webQDA; Futuros Maestros; Matemáticas; Educación Matemática.

\section{Educational Robotics to Teach Mathematics: Opinions of the Students of the Degree in Primary Education}

\begin{abstract}
Over the years, educational robotics has gained more weight, being increasingly present in the teaching and learning process. The use of robotics as an educational resource is important due to the benefits that it can cause among students, such as increased attention, motivation, curiosity, etc., as can be observed in the different studies analysed for this research. More and more authors are studying the benefits or damages that educational robotics can have in the teaching and learning process of students. This research will analyse the opinions of future primary school teachers on the use of robotics as an educational resource to teach mathematics. The opinions of fifty students of the Degree of Primary Education of the University of Extremadura (Badajoz, Spain) will be analysed. The data is obtained through an open question through a forum and, in order to carry out the pertinent analyses, the WebQDA software was used (Neri, Costa and Moreira, 2011). With WebQDA, the different opinions of the students were coded, according to a category system designed by Soto-Ardila, Caballero, Melo and Luengo (2019). The results show that, for the majority of the students in the sample, the use of robotics can provide numerous benefits in the teaching learning process in general and, more specifically, in the teaching and learning of mathematics, these being benefits, greater than the disadvantages that its use can cause.
\end{abstract}

Keywords: Educational Robotics; webQDA; Future Teachers; Mathematics; Mathematics Education. 


\section{Introducción del Estudio}

La importancia de la robótica educativa en los contextos educativos va siendo cada vez mayor, convirtiéndose en una herramienta muy útil para el proceso de enseñanzaaprendizaje (González, Flores y Muñoz, 2021). A pesar de ello, aún no se encuentra enmarcada en el currículum de primaria, algo que le permitiría convertirse en un recurso "formal". Esto, hace que sea necesario que se produzca un cambio en las aulas, con el fin de que los alumnos se conviertan en participantes en su propio proceso de enseñanza y aprendizaje, y no en meros oyentes y repetidores. El profesor a su vez, pasará de ser un transmisor de información a ser un guía que ayude a los alumnos con los problemas que puedan surgirles durante el proceso de aprendizaje (Barrera, 2015).

Sin embargo, a pesar de ello, muchas son las personas que emplean la robótica educativa como recurso didáctico, haciendo que los robots sean recursos útiles para la enseñanza de muchos contenidos como, por ejemplo, los matemáticos.

Bravo y Forero (2012) indican la importancia de introducir la robótica educativa de forma adecuada en el currículo, dado que, el uso de los robots permite desarrollar diversas competencias y habilidades, así como varias destrezas que permitan al alumnado superar diversos retos.

Otros autores como Márquez y Ruiz (2014), indican que, el uso de la robótica fomenta la motivación y la creatividad, permitiendo que los alumnos desarrollen, con el paso del tiempo, ciertas habilidades manuales y cognitivas. Del mismo modo que Márquez y Ruiz (2014), los autores Moreno, Muñoz, Serracín, Quintero, Pitti y Quiel (2012), en su investigación, destacan que la robótica educativa permite, no solo motivar, sino, además, facilitar conocimientos a los alumnos. Consideran que, utilizando la robótica, los alumnos pueden llegar a aprender conceptos más abstractos y complejos de una forma más sencilla, lúdica y motivadora.

\subsection{Importancia de la Robótica Educativa: Ventajas e Inconvenientes}

Antes de mencionar investigaciones que aborden la importancia de la robótica educativa, resulta necesario definir qué es la robótica educativa. Ruiz-Velasco (2007), define la robótica educativa como "aquella disciplina que, mediante el desarrollo y diseño de robots, los estudiantes se introducen en el estudio de las ciencias y de la tecnología".

Otros autores como Bravo y Forero (2012), la definen como "aquel recurso que permite que el aprendizaje de los alumnos sea más fácil, ayudando al profesor en el proceso de enseñanza. También permite potenciar aspectos como la socialización, la iniciativa o la creatividad".

Existen numerosas investigaciones sobre la importancia que la robótica puede tener en el contexto educativo. Merino, Villena, González y Cózar (2017), por ejemplo, consideran que, dentro de la tecnología educativa, la robótica se convierte en uno de los recursos que presentan una mayor importancia, permitiendo aportar un aprendizaje constructivo para el alumno.

Ruiz (2007) explica la importancia que la robótica educativa presenta en las escuelas, ya que es un recurso que permite ocasionar cambios significativos en el aprendizaje de los alumnos. Estos cambios son posibles gracias a la interdisciplinariedad que la robótica educativa presenta, así como, al desarrollo de ciertas habilidades que, probablemente, los alumnos no habían trabajado previamente (Papert, 1980; García y Reyes, 2012).

Otros autores consideran que, seleccionar un material o recurso puede llegar a ser fundamental para que los alumnos comprendan mejor un contenido. Esto se debe a que, dependiendo del material, los alumnos pueden llegar a estar más motivados, convirtiéndose esto en una ventaja fundamental para mejorar su aprendizaje (Cabero, Fernández y Marín, 2017; Merino, Villena, González y Cózar, 2017). 
Morales (2017), en concordancia con Hernández (2016) y Fernández (2006), considera que la robótica educativa resulta importante ya que tiene como ventaja el fomentar el trabajo cooperativo, consiguiendo que los alumnos se relacionen y aprendan a trabajar en equipo. También considera que los alumnos irán desarrollando diversos procesos cognitivos y sociales que les permitirán aprender de forma significativa.

Sin embargo, existen otros autores que destacan que la robótica educativa presenta alguna que otra desventaja. Es el caso de Vega-Moreno, Cufié, Rueda y Llinás (2016), quienes afirman que la robótica educativa resulta inaccesible para muchas personas. Alguno de los motivos por los que ocurre esto, es por el precio tan caro de los materiales, haciendo que no sea fácil conseguir kits de robótica.

También se puede decir que, aunque hay muchas empresas que pretenden trabajar la robótica educativa como actividades extraescolares, estas no siempre son accesibles para los niños, puesto que, el precio de las inscripciones, en muchos casos, es elevado, impidiendo acceder a aquellos alumnos menos favorecidos (Soto-Ardila, Caballero, Melo y Luengo, 2019).

\section{Objetivo y Preguntas de Investigación}

El presente estudio surge como resultado de las inquietudes investigadoras de los participantes del mismo, inquietudes que pueden traducirse en varias preguntas de investigación:

- ¿Qué opinan los futuros maestros sobre la robótica educativa?

- ¿Cuáles son las ventajas, según los estudiantes del grado, que puede tener el utilizar la robótica educativa como recurso didáctico?

- ¿Conocen las desventajas que puede tener la robótica educativa?

- ¿Los robots son accesibles para toda la población?

Tras el planteamiento de las preguntas de investigación, se destacó el siguiente objetivo: "Analizar las opiniones que los estudiantes del Grado de Educación Primaria (tercer curso) presentan sobre el uso de la robótica educativa como herramienta para la enseñanza de las matemáticas, analizando los inconvenientes o las ventajas que puede tener su utilización".

\section{Metodología}

Este estudio se enmarca dentro de un diseño fenomenológico de investigación, a través del cual, se podrán analizar las experiencias personales de cada uno de los participantes para obtener unas conclusiones finales.

La muestra seleccionada para llevar a cabo esta investigación fueron 50 alumnos, de tercer curso del Grado de Educación Primaria, de la Universidad de Extremadura.

Dichos alumnos tuvieron la oportunidad de trabajar con robots con anterioridad, y han podido observar las posibilidades o las dificultades que tienen a la hora de emplearse como recursos para enseñar matemáticas.

En las prácticas realizadas con robots, los alumnos emplearon el kit "LEGO Education WeDo 2.0", con el que pudieron trabajar diversos aspectos como el montaje de los robots y la programación de los mismos para desarrollar diversas actividades.

Tras la experiencia con los robots, los alumnos realizaron una reflexión sobre las opiniones que presentan con respecto al uso de la robótica educativa en contextos escolares. 
Dicha reflexión, se inició con una pregunta impulsora a través de un foro del campus virtual de la Universidad de Extremadura: "Haz una reflexión sobre cuáles son las ventajas o inconvenientes que, el uso de la robótica, puede tener en el ámbito educativo. Indica, además, la utilidad que crees que puede tener para el proceso de enseñanza/aprendizaje de las matemáticas".

Una vez realizada la pregunta y obtenidas las respuestas de los alumnos, se realizó un proceso de depuración, sistematización y análisis de todos los datos recabados. Los pasos seguidos para ello fueron los siguientes (Soto-Ardila, Melo, Caballero y Luengo, 2019):

En primer lugar, se procedió a la identificación de lo que se denominan "unidades de información". Tras ello, se realizó una categorización de dichas unidades (teniendo en cuenta un sistema de categorías previo que se explicará a continuación).

Una vez categorizadas, se procedió a la codificación de dichas unidades de información. Posteriormente, se observó si había alguna categoría diferente a la del sistema y, en caso de haberla, se añadiría al sistema de categorías. Finalmente, se analizaron las unidades de información.

El sistema de categorías empleado para la investigación fue el siguiente:

Tabla 1. Adaptación del sistema de categorías elaborado por Soto-Ardila, Caballero, Melo y Luengo (2019).

\begin{tabular}{ll}
\hline \multicolumn{1}{c}{ Dimensiones } & \multicolumn{1}{c}{ Categorías } \\
\hline & A1. Desarrollo de la creatividad e imaginación. \\
A2. Desarrollo del pensamiento crítico, lógico y la \\
resolución de problemas. \\
A3. Fomenta la libertad de expresión. \\
A4. Herramientas eficaces para comprender mejor los \\
$\begin{array}{l}\text { A. Ventajas de la Robótica } \\
\text { contenidos (aprendizaje significativo). }\end{array}$ \\
A5. Desarrollo de habilidades (observación). \\
A6. Enseñanza de competencias transversales. \\
A7. Enseñanza motivadora, lúdica, divertida, \\
entretenida. \\
A8. Desarrollar habilidades motoras. \\
B1. Difícil acceso al material (precios y mantenimiento \\
caro). \\
B2. Errores al desarrollar las prácticas con robots (por \\
ejemplo, problemas al conectar los robots con el \\
ordenador). \\
B3. Desfase ocasionado por los robots (por ejemplo, \\
Robótica Educativa
\end{tabular}

Como se puede observar en la tabla anterior, el sistema de categorías está formado por dos dimensiones fundamentales: ventajas de la robótica educativa y desventajas de la robótica educativa. A su vez, dentro de cada dimensión se pueden encontrar trece categorías (ocho para la categoría de ventajas y cinco para la categoría de desventajas).

Este sistema de categorías sirvió para la realización del análisis pertinente a través del software de investigación cualitativa WebQDA (Neri, Costa y Moreira, 2011), software online que permite el trabajo colaborativo desde cualquier dispositivo y lugar. 


\section{Resultados Obtenidos}

Los resultados obtenidos en esta investigación pretenden resolver el objetivo general planteado previamente: "Conocer y analizar las opiniones que los estudiantes del Grado de Educación Primaria (tercer curso) presentan sobre el uso de la robótica educativa como herramienta para la enseñanza de las matemáticas, analizando los inconvenientes o las ventajas que puede tener su utilización".

A la hora de estructurar los resultados, en primer lugar, se abordaron los datos obtenidos de la primera dimensión (ventajas de usar la robótica educativa) y, posteriormente, de la segunda dimensión (desventajas de usar la robótica educativa).

En relación a las ventajas se obtuvieron los siguientes resultados:

Fig. 1. Resultados de la primera dimensión.

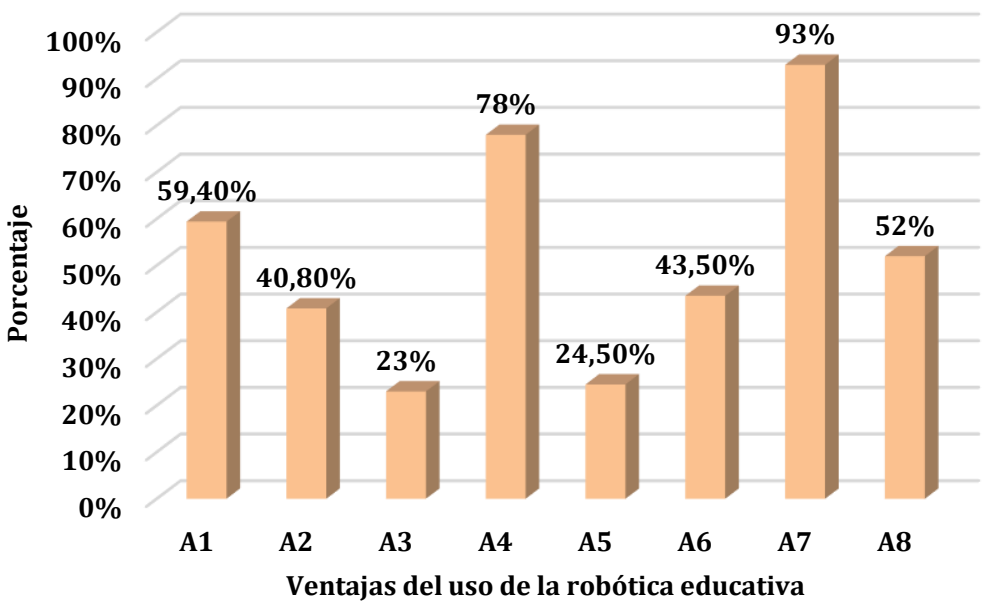

Como se puede observar en la figura anterior, la mayor parte de la muestra $(93 \%$ de los participantes) consideran que una de las ventajas fundamentales de la robótica educativa es la A7 "Enseñanza motivadora, lúdica, divertida, entretenida", seguida de la ventaja A4 "Herramientas eficaces para comprender mejor los contenidos (aprendizaje significativo)", la cual fue mencionada por el $78 \%$ de los alumnos de la muestra. Algunos ejemplos de estas dos ventajas, sacado de los textos analizados, se pueden mostrar a continuación:

[...] Con los robots los alumnos podrían aprender de una forma lúdica, motivándoles mucho más que de una forma tradicional [...].

[...] Probablemente, empleando la robótica educativa, los alumnos pueden llegar a comprender mejor los conceptos a trabajar [...].

[...] Nuestros alumnos pueden divertirse más si se utiliza la robótica educativa, llegando a aprender mejor los contenidos y a estar más motivados $[\ldots]$.

[...] Los alumnos pueden aprender mucho mejor los contenidos, llegando a interiorizarlos y convertirlos en un aprendizaje significativo [...].

El $59,4 \%$ y el $52 \%$ de los alumnos de la muestra, se enmarcaban en las categorías A1 "Desarrollo de la creatividad e imaginación" y A8 "Desarrollar habilidades motoras", respectivamente. Algunos ejemplos de ello son:

[...] El hecho de utilizar piezas de lego, por ejemplo, puede fomentar la imaginación de los alumnos, así como su creatividad, ya que tienen que pensar e imaginar qué pueden construir [...]. 
Vol. 7 | Investigación Cualitativa en Educación: Avances y Desafíos

[...] El trabajo manual que tienen que hacer, a la hora de construir robots, hace que desarrollen otras habilidades, como puede ser el caso de las habilidades motoras o la coordinación ojo-mano [...].

Para el $43,5 \%$ y el $40,8 \%$ de los estudiantes de la muestra, las principales ventajas son: A6 "Enseñanza de competencias transversales" y A2 "Desarrollo del pensamiento crítico, lógico y la resolución de problemas". Estos fueron algunos de los ejemplos encontrados:

[...] El uso de los robots fomenta el desarrollo de actividades transversales, puesto que se pueden aplicar en diferentes disciplinas, desarrollando diversas competencias [...].

[...] La lógica puede trabajarse con la robótica educativa, así como el pensamiento crítico. Además, puede ser útil para trabajar la resolución de problemas, entre otros aspectos [...].

Las últimas ventajas que se encontraron entre las distintas reflexiones fueron las correspondientes a las categorías A5 "Desarrollo de habilidades (observación)" (24,5\%) y A3 "Fomenta la libertad de expresión" (23\%). Ejemplos de ello son:

[...] Se puede desarrollar, habilidades como la observación, entre otras, haciendo que sea un recurso útil para el alumnado [...].

[...] Otra ventaja es que, además de estimular aspectos como la imaginación, fomenta la libertad de expresión, haciendo que los alumnos sean más creativos y versátiles [...].

En relación a las desventajas de utilizar la robótica educativa, los resultados obtenidos fueron los siguientes:

Fig. 2. Resultados de la segunda dimensión.

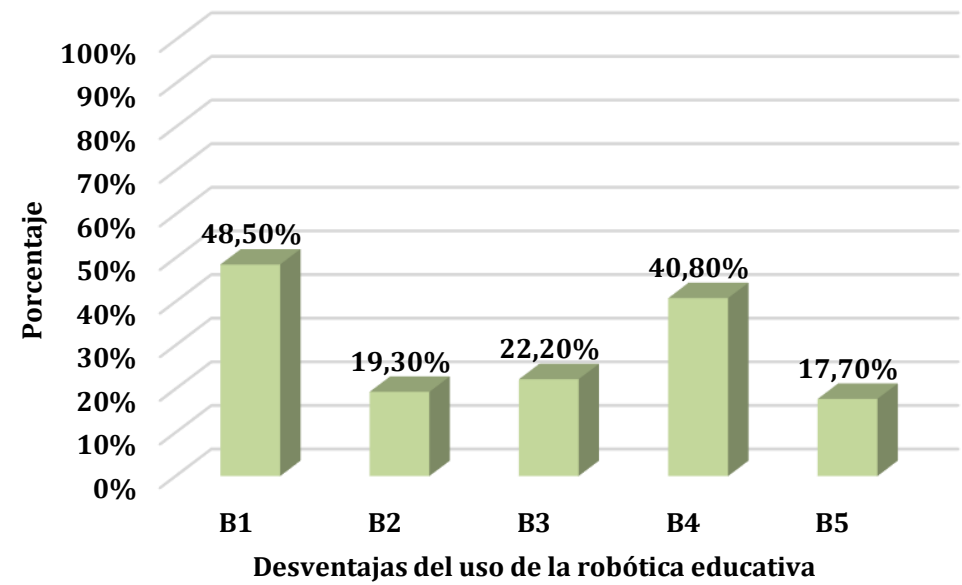

Como se puede observar en la figura 2, la mayor parte de los alumnos de la muestra $(48,5 \%)$ consideran que la principal desventaja es B1 "Difícil acceso al material (precios y mantenimiento caro)", seguida de la B4 "Requiere de una mayor formación del docente" $(40,8 \%)$. Estos son algunos fragmentos de los relatos de los alumnos:

[...] Un kit de lego, por ejemplo, te puede costar entre $100 €$ y $300 €$, con lo que no todo el mundo puede comprárselo [...].

[...] Ahora claro, si tú quieres emplear la robótica, tienes que dedicarle mucho tiempo a aprender a utilizar los robots, diseñar la clase, los contenidos a trabajar, etc. [...]. 
La siguiente desventaja que ha obtenido un mayor porcentaje es la B3 "Desfase ocasionado por los robots (por ejemplo, exactitud a la hora de hacer giros)" $(22,2 \%)$, con lo que se puede decir que, para algunos de los estudiantes del grado, los robots no son tan exactos como parecen, pudiendo ocasionar problemas a la hora de trabajar conceptos matemáticos, como pueden ser los ángulos, giros, distancias, etc. Algunos fragmentos de los relatos de los alumnos fueron los siguientes:

[...] Como hemos podido comprobar con nuestra propia experiencia, los robots de LEGO al realizar un giro, suele girar más de la cuenta debido a la propia inercia del robot [...].

[...] Me he podido dar cuenta de que siempre que se le da una orden al robot, hay que pensar en el pequeño desfase que tiene a la hora de realizarla, por ejemplo, cuando quiero que recorra una distancia, he visto que tengo que indicarle que avanza un poco más para que llegue al sitio que quiero, no coincidiendo con la "medida real" que debería de recorrer [...].

Otra buena parte de la muestra, considera que los robots pueden dar algunos que otros problemas de conectividad, pudiendo ocasionar una pérdida de tiempo a la hora de impartir la asignatura. Por ello, el $19,3 \%$ de los alumnos tuvieron en cuenta la desventaja B2 "Errores al desarrollar las prácticas con robots (por ejemplo, problemas al conectar los robots con el ordenador)". Estos son algunos ejemplos de los relatos:

[...] Considero que los robots pueden ser una pérdida de tiempo y más si no tienes unos equipos adecuados para conectarlos [...].

[...] Con respecto a algunos robots como el LEGO WeDo 2.0, necesita un tipo de Bluetooth determinado $y$, por desgracia, solo los ordenadores más modernos lo tienen, con lo que no todo el mundo puede conectarlo a su equipo $[\ldots]$.

Finalmente, para el $17,7 \%$ de los alumnos de la muestra, una desventaja importante es la B5 "Puede distraer a los alumnos, alborotando la clase", ya que consideran que, una clase en la que se utiliza la robótica, mal gestionada, puede llegar a ser un caos, ocasionando más problemas que beneficios. Algunos ejemplos fueron:

[...] Hay que saber cómo vas a realizar la actividad si no quieres que el usar la robótica se convierta en un arma de doble filo. [...].

[...] Tienes que tener claro que, los robots son llamativos para los niños y, el usarlos implicará ruido y alboroto por parte de ellos, y esto puede ser negativo ya que se pueden distraer más de la cuenta y no atender a las explicaciones $[\ldots]$.

\section{Conclusiones y discusión}

Para concluir la presente investigación, es importante recordar el objetivo que planteamos al principio: "Conocer y analizar las opiniones que los estudiantes del Grado de Educación Primaria (tercer curso) presentan sobre el uso de la robótica educativa como herramienta para la enseñanza de las matemáticas, analizando los inconvenientes o las ventajas que puede tener su utilización".

Como se ha podido observar, dicho objetivo se ha cumplido, puesto que, a lo largo del estudio, se han ido analizando las distintas opiniones de los alumnos de la muestra, pudiendo sacar las ventajas y desventajas que, el uso de la robótica educativa, pueden tener.

Entre las ventajas que se pueden obtener empleando la robótica educativa, pudimos ver como la motivación o el trasfondo lúdico, entretenido y divertido tuvieron un gran peso en nuestro estudio, coincidiendo con lo que autores como Moreno, Muñoz, Serracín, Quintero, Pitti y Quiel (2012) afirman. 
Autores como Ruiz (2007) o Bravo y Forero (2012), mencionan la importancia de la robótica educativa para la consecución de un aprendizaje significativo, algo que es fundamental para el desarrollo de los alumnos. En nuestra investigación hemos podido observar que, para los alumnos de la muestra, ésta es una de las ventajas más importantes ya que conseguir que los alumnos aprendan de forma significativa, resulta vital para su formación.

Con respecto a las principales desventajas del uso de la robótica educativa, los alumnos de la muestra hacen hincapié a la dificultad de acceso a los materiales debido a los altos precios de éstos, así como las elevadas tarifas de las empresas que se dedican a la enseñanza de la robótica educativa. Todo esto concuerda con las investigaciones de Vega-Moreno, Cufí, Rueda y Llinás (2016).

Para finalizar, resulta necesario hacer una mención especial al empleo de las metodologías cualitativas para la realización de este tipo de investigaciones, puesto que, gracias a estos métodos, se pueden analizar grandes cantidades de datos llegando al trasfondo de los mismos, ya que, no solo obtenemos porcentajes y datos numéricos si no que, además, podemos saber detalladamente cuáles son las opiniones reales de la muestra y el porqué de esas opiniones.

\section{Agradecimientos}

Este trabajo ha sido financiado por el Fondo Europeo de Desarrollo Regional y Junta de Extremadura (Proyecto GR18115).

\section{JUNTA DE EXTREMADURA}

Consejería de Economía, Ciencia y Agenda Digital

\section{Referencias}

Barrera, N.L. (2015) Uso de la robótica educativa como estrategia didáctica en el aula. Revista Praxis \& Saber 11(6), 215-234.

Bravo, F., y Forero, A. (2012). La robótica como un recurso para facilitar el aprendizaje y desarrollo de competencias generales. Education in the knowledge society, 13(2), 120-136.

Cabero, J., Fernández, B., y Marín, V. (2017). Dispositivos móviles y realidad aumentada en el aprendizaje del alumnado universitario. RIED. Revista Iberoamericana de Educación a Distancia, 20(2), 167-185.

Fernández, A. (2006). Metodologías activas para la formación de competencias. Educatio siglo $X X I, 24,35-56$.

García, Y., y Reyes, D. (2012). Robótica educa- tiva y su potencial mediador en el desarrollo de las competencias asociadas a la alfabetización científica. Revista Educación y Tecnología, 2, 42-55.

González, M. O., Flores, Y. A., y Muñoz, C. (2021). Panorama de la robótica educativa a favor del aprendizaje STEAM. Revista Eureka Sobre Enseñanza Y Divulgación De Las Ciencias, 18(2), 1-19.

Hernández, V. (2016). Robótica educativa, roboti ¿qué? (Trabajo de Fin de Grado). Universidad de La Laguna, Tenerife, España.

Márquez, J. E. y Ruíz, J. H. (2014). Robótica educativa aplicada a la enseñanza básica secundaria. Revista DIM, 30, 1-12.

Merino, J., Villena, R., González J., y Cózar, R. (2017). Análisis del efecto de la robótica en la motivación de estudiantes de tercero de educación primaria durante la resolución de tareas de interpretación de planos. Revista de Estudios y Experiencias en Educación, 3, 163-173. 
Vol. 7 | Investigación Cualitativa en Educación: Avances y Desafíos

Morales, P (2017). La robótica educativa: una oportunidad para la cooperación en las aulas. En Ruiz, J., Sánchez, J. y Sánchez, E. (Edit.). Innovación docente y uso de las TIC en educación. Málaga: UMA Editorial.

Moreno, I., Muñoz, L., Serracín, J. R., Quintero, J., Pittí, K. y Quiel, J. (2012). La robótica educativa, una herramienta para la enseñanza-aprendizaje de las ciencias y las tecnologías. TESI-Teoría de la Educación, Educación y Cultura en la Sociedad de la Información, 13(2), 74-90.

Neri, F., Costa, A. P. y Moreira, A. (2011). Análise de Dados Qualitativos Suportada pelo Software webQDA, Atas da VII Conferência Internacional de TIC na Educação: Perspetivas de Inovação (CHALLANGES2011), pp. 49-56, 2011.

Papert, S. (1980). Mindstorms: Children, computers, and powerful ideas. New York: Basic Books.

Ruiz-Velasco, E. (2007). Educatrónica. Innovación en el aprendizaje de las ciencias y la tecnología. Buenos Aires, Argentina, Ediciones Díaz de Santos.

Soto-Ardila, L. M., Melo, L., Caballero, A. y Luengo, R. (2019). Estudio de las opiniones de los futuros maestros sobre el uso de los videojuegos como recurso didáctico a través de un análisis cualitativo. RISTI - Revista Ibérica de Sistemas e Tecnologías de Informaçao, 33, 48-63. DOI: 10.17013/risti.33.48-63

Vega-Moreno, D., Cufié, X., Rueda, Mạ . J, \& Llinás, D. (2016). Integración de robótica educativa de bajo coste en el ámbito de la educación secundaria para fomentar el aprendizaje por proyectos. International Journal of Educational Research and Innovation (IJERI), 6, 162-175. 